\title{
Huge Osteoma on Suboccipital Region: A Case Report
}

\author{
Ji-Hoon Park ${ }^{1}$, Hyuk-Jin Oh ${ }^{1}$, Dong-Gyu Yeo ${ }^{2}$, Jae-Sang Oh${ }^{1}$, Seok-Mann Yoon ${ }^{1}$, Jai-Joon Shim ${ }^{1}$ \\ ${ }^{1}$ Department of Neurosurgery, Soonchunhyang University Cheonan Hospital, Cheonan, \\ ${ }^{2}$ Department of Neurosurgery, Soonchunhyang University Gumi Hospital, Gumi, Republic of Korea
}

Corresponding author:

Jai-Joon Shim

Department of Neurosurgery,

Soonchunhyang University Cheonan

Hospital, 31, Suncheonhyang 6-gil,

Dongnam-gu, Cheonan 31151,

Republic of Korea

Tel: +82-41-570-3650

Fax: +82-41-572-9297

E-mail: jjshim@sch.ac.kr

Received: November 18, 2018

Revised: December 29, 2018

Accepted: February 10, 2019
Osteoma is the most common benign tumor in the craniofacial bone. We present a case of osteoma, a rare site in a patient with palpable mass on suboccipital area. A 54-year-old woman presented with palpable mass in the right occipital area. Brain computed tomography scan indicated that the mass was starting from the outer table of the occipital bone. The mass was separated well from the cortical bone. Histopathology report presented osteoma diagnosis composed of a compact bone. After the operation, the patient was discharged without any complications and follow-up was done for 8 years without incidences of recurrence. It is very rare for osteoma to occur in the occipital bone. We reported a case of a huge osteoma of the occipital bone that grew without invasion of the inner table of the cranial bone.

Key Words: Osteoblastoma; Osteoma; Skull neoplasms

\section{INTRODUCTION}

Osteoma is the most common benign tumor in the craniofacial bone ${ }^{()}$. It is mostly seen in the frontal sinus, and occurs in the order of ethmoid, maxillary and sphenoid sinus. Tumor growth is so slow and it is predominantly asymptomatic until a certain size is attained and it is often found on simple skulls. If the size of the tumor is large, it may cause clinical symptoms such as severe headache and dizziness. We report a case of osteoma, a rare site in a patient with palpable mass on the suboccipital area.

\section{CASE REPORT}

A 54-year-old woman presented with palpable progressively growing mass on the right occipital area. The patient felt neck pain, which slightly increased over a period of 40 years. However, she did not experience any headaches or dizziness. In addition, she had no history of head trauma or cranial disease. Physical examination showed no change in tenderness, heating sensation, or color change. Besides, there was no limitation on the neck movement. After a skull X-ray, a $51 \times 45 \times 40 \mathrm{~mm}$ sized radiopaque mass with a rounded border was observed on the right occipital area (Fig. 1). Brain computed tomography (CT) scan showed masses starting from the outer table of the occipital bone. Most of the mass consisted of cortical bone but there was no lesion enhancing into the mass nor destruction of the inner table. Under general anesthesia, the neck was flexed using a horseshoe as a prone position, an oblique incision was made around the mass, and muscle dissection was performed to expose the osteoma. The mass was separated from the cortical bone using an osteotome and the margins were trimmed using an electric drill. Histopathology report indicated osteoma diagnosis composed of compact bone (Fig. 2). After the operation, the patient was discharged without any complications and was followed up was done for 8 years without recurrence.
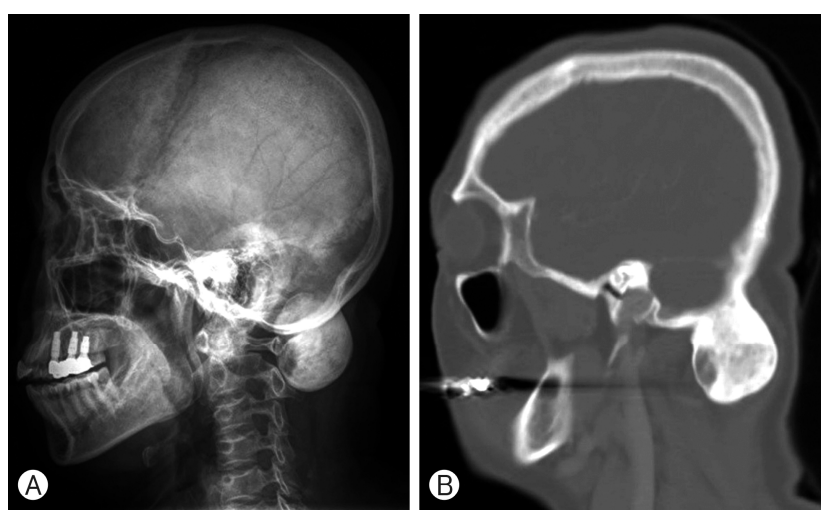

Fig. 1. (A, B) Radiological findings. Ovoid $51 \times 45 \times 40 \mathrm{~mm}$ sized well-defined bony mass lesion is observed. The mass originated from the cortex of the right occipital bone. 


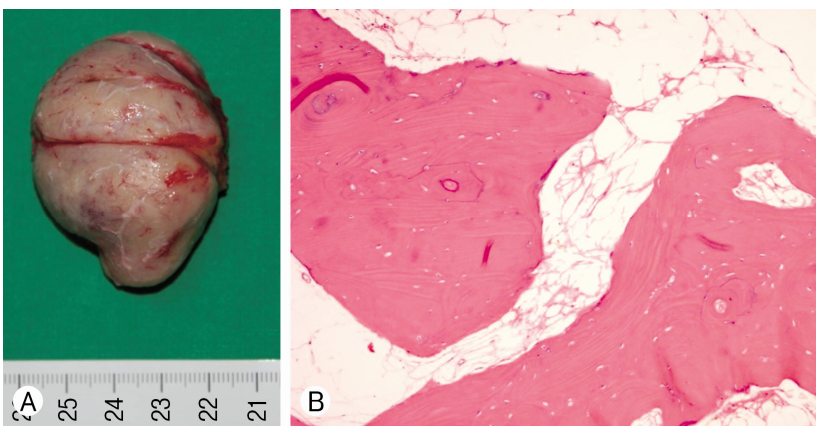

Fig. 2. (A) Gloss specimen of the bony tumor and (B) histological findings. Hematoxylin and eosin stain showed an irregular trabeculae of woven bone and surrounding the fibrovascular stroma.

\section{DISCUSSION}

Osteoma is a benign tumor that mostly occurs in the craniofacial bone, within the frontal sinus, ethmoid, maxillary and sphenoid sinus. It is frequently found in adults during the first 3 decades of their lives and it is mainly prevalent in women ${ }^{7}$. An osteoma more than $3 \mathrm{~cm}$ in size is classified as a giant osteoma, and the few cases reported on giant osteomas involve the occipital regions ${ }^{4,10,11)}$. It is histologically divided into ivory, mature and mixed type ${ }^{12)}$. The ivory type is composed of hard and compact bone with almost no fibrovascular interstitial tissues. It mainly occurs in the frontal sinus and has a slow growth rate. On the other hand, the mature type is composed of mature cancellous bone and mainly occurs in the maxillary sinus and ethmoid sinus. Mature type of tumor grows faster than the ivory type ${ }^{3)}$. Mixed osteoma is a mixture of ivory and mature type. In the present case, the CT scan indicated that osteomas were thought to be ivory type, consisting of a compact bone. Histologically, a compact composition was the main finding, so this case confirmed to be an ivory osteoma.

The developmental hypothesis, which assumed the site of osteoma is the junction of the frontal bone (membranous) and the cartilaginous bone (cartilaginous bone) was proposed. The developmental, traumatic, and infectious causes of osteomas hypotheses were presented, but none of them had exact findings ${ }^{1,5,9,14)}$. They were deemed to be reliable markers for the early detection of Gardner syndrome ${ }^{8,10)}$. However, no specific etiology was confirmed in this case.

The diagnosis of osteoma is relatively simple because the radiopacity of plain radiography is depicted to be very useful for diagnosing osteoma. Brain CT shows a smooth, well defined and a homogeneous mass, which is also helpful in the diagnosis and treatment of the osteoma. Brain CT with 3-dimensional reconstruction can be used to plan the surgery because of the relationship between the osteoma and the surrounding bone structures, which can easily be illuminated. On the other hand, magnetic resonance imaging shows a good relationship between osteoma and intracranial structures, but its usefulness is limited in its relation to surrounding bone structures, since osteoid structures exhibit signal avoidance ${ }^{3)}$. Differential diagnosis of osteoma including benign bone-forming lesions and malignant lesions such as osteosarcoma and osteoblastic metastasis should be considered ${ }^{12)}$.

Surgical resection is the best treatment for osteomas. However, if the lesion is small or asymptomatic, regular radiological image follow-up is possible. Surgery is mainly is considered if osteoma causes pain or has neurological symptoms. Surgery can also be performed for cosmetic improvement ${ }^{10)}$.

Surgical resection should be done to completely outline the bones of all normal cortex around the lesion. Generally, the osteoma of the skull is treated according to the location and size of the mass. Craniotomy and cranioplasty may be necessary after removal of the mass using a drill if the mass invades the inner table. If the lesion is confined to the external cortex, it is not difficult to find the interface between the osteoma and the normal bone. In addition, total resection is possible if the recurrence rate is very low ${ }^{2,13}$.

\section{CONCLUSION}

It is very rare for osteoma to occur in the occipital bone. We reported a case of a huge osteoma of the occipital bone that grew without invasion of the inner table of the cranial bone.

\section{CONFLICT OF INTEREST}

No potential conflict of interest relevant to this article was reported.

\section{REFERENCES}

1. Al-Sebeih K, Desrosiers M: Bifrontal endoscopic resection of frontal sinus osteoma. Laryngoscope 108:295-298, 1998

2. Birrell JF: Osteoma of the mastoid. J R Coll Surg Edinb 23:305309, 1978

3. Eom KS, Kim TY, Kim JM: Giant osteoma of the anterior cranial fossa: Case report. J Korean Neurosurg Soc 32:59-62, 2002

4. Erten F, Hasturk AE, Pak I, Sokmen O: Giant occipital osteoid osteoma mimicking calcified meningioma. Neurosciences (Riyadh) 16:363-365, 2011

5. Gossios K, Bai M, Psilas K: Giant aggressive osteoma of the frontal sinus. Clin Radiol 54:193-196, 1999

6. Haddad FS, Haddad GF, Zaatari G: Cranial osteomas: their classification and management. Report on a giant osteoma and review of the literature. Surg Neurol 48:143-147, 1997

7. Ito K, Sugawara T, Tamura K, Kawabata S, Kobayashi D, Maehara T: Occipital condyle osteoid osteoma with severe occipital pain that disappeared after surgical resection. NMC Case Rep 
J 2:128-131, 2015

8. Koh KJ, Park HN, Kim KA: Gardner syndrome associated with multiple osteomas, intestinal polyposis, and epidermoid cysts. Imaging Sci Dent 46:267-272, 2016

9. Mansour AM, Salti H, Uwaydat S, Dakroub R, Bashshour Z: Ethmoid sinus osteoma presenting as epiphora and orbital cellulitis: case report and literature review. Surv Ophthalmol 43:413426, 1999

10. Munakomi S, Bhattarai B: A rare case report on suboccipital region benign giant osteoma. Case Rep Neurol Med 2016:
2096701, 2016

11. Nishitani K, Okajima K, Tsuda T: A case of giant osteoma in the occipital bone. No Shinkei Geka 18:947-952, 1990

12. Park SJ, Kim YH: A case of giant osteoma developed from the mastoid cortical bone. Korean J Audiol 16:95-98, 2012

13. Probst LE, Shankar L, Fox R: Osteoma of the mastoid bone. J Otolaryngol 20:228-230, 1991

14. Summers LE, Mascott CR, Tompkins JR, Richardson DE: Frontal sinus osteoma associated with cerebral abscess formation: a case report. Surg Neurol 55:235-239, 2001 